\title{
Integrating Learning, Practice and Professionalism: The Nottingham 5 year
}

MPharm

\section{Author: Tom Gray, School of Pharmacy, University of Nottingham, Nottingham}

Background: Following recommendations from the Modernising Pharmacy Careers Board for 'one five-year curriculum that is jointly owned, planned and delivered by universities and employers, where students have greater access to work-based learning and contact with patients from earlier in degree programmes', University of Nottingham designed a 5 year MPharm programme that 'builds on the strong scientific foundation...to enhance clinical skills and the attitudes, values and behaviours required to deliver effective care and treatments' ${ }^{1}$. This novel programme received Step 1 accreditation by the General Pharmaceutical Council in 2013, integrating two 6 month professional placements into years 4 and 5 of the MPharm. Years 1-3 involve a spiral curricular approach to learning, integrating science and practice, through combined 'drug, medicine and patient' modules, supported by a zero credit professional competencies module, to establish the attitudes, values and professional behaviours required for practice. Students will undertake their first professional placement in August 2015.

Description of work: Work is ongoing to establish valid, reliable and consistent assessment of the professional placements in a safe, supported, supervised and quality assured training environment. This is being done in partnership with an Advisory Board, consisting of employers, regional training providers and commissioners, to build on existing regional training and quality frameworks, and match student preferences to employer and future workforce needs. Academic facilitators, working in collaboration with pre-registration training tutors will support, assess and quality assure training.

Proposed evaluation: Evaluation will be based on a portfolio of evidence developed using established formative assessment methods and supervised learning events, tested for reliability and consistency across sectors, along with audit and online summative assessment. Independent educationalists will support evaluation of educational and professional outcomes. A survey of stakeholders, demonstrates enthusiasm and support for the new course to better meet the needs of patients, employers and future pharmacists.

\section{References}

1. Pharmacist student numbers and reform of undergraduate education and preregistration training in England. Progress update for MPC Professional Board. Department for Health. Dec 2012 